\title{
Gray-Related Support Vector Machine Optimization Strategy and Its Implementation in Forecasting Photovoltaic Output Power
}

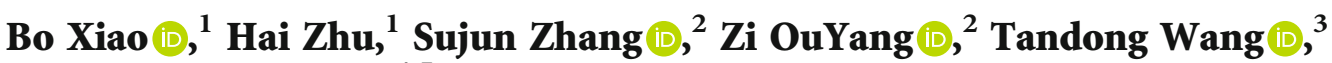 \\ and Saeed Sarvazizi iD ${ }^{4,5}$ \\ ${ }^{1}$ School of Electronic and Electrical Engineering, Shanghai University of Engineering Science, Shanghai 201620, China \\ ${ }^{2}$ Meteocontrol (Shanghai) Data Tech Co., Ltd, Shanghai 200233, China \\ ${ }^{3}$ School of Electronics and Information, Northwestern Polytechnical University, Xi'an, Shaanxi 710072, China \\ ${ }^{4}$ Department of Petroleum Engineering, Ahwaz Faculty of Petroleum Engineering, Petroleum University of Technology (PUT), \\ Ahwaz, Iran \\ ${ }^{5}$ Department of Petroleum Engineering, Amirkabir University of Technology (Tehran Polytechnic), Tehran, Iran
}

Correspondence should be addressed to Bo Xiao; 02180013@sues.edu.cn and Saeed Sarvazizi; sarvazizi.saeed@aut.ac.ir

Received 26 November 2021; Revised 2 February 2022; Accepted 11 February 2022; Published 25 February 2022

Academic Editor: Hafiz Muhammad Ali

Copyright (C) 2022 Bo Xiao et al. This is an open access article distributed under the Creative Commons Attribution License, which permits unrestricted use, distribution, and reproduction in any medium, provided the original work is properly cited.

\begin{abstract}
Reliable and accurate photovoltaic (PV) output power projection is critical for power grid security, stability, and economic operation. However, because of the indirectness, unpredictability, and solar energy volatility, predicting precise and reliable photovoltaic output power is a complicated subject. The photovoltaic output power variable is evaluated in this study using a powerful machine learning approach called the support vector machine model based on gray-wolf optimization. A vast dataset of previously published papers was compiled for this purpose. Several studies were carried out to assess the suggested model. The statistical evaluation revealed that this model predicts absolute values with reasonable accuracy, including $R^{2}$ and RMSE values of 0.908 and 74.6584, respectively. The practical input data were also subjected to sensitivity analysis. The results of this analysis showed that the air temperature parameter has a greater effect on the target parameter than the solar irradiance intensity parameter (relevancy factor equal to 0.75 compared to 0.49 , respectively). The leverage approach was also used to test the accuracy of actual data, and the findings revealed that the vast majority of data is accurate. This basic but accurate model may be quite effective in predicting target values and could be a viable substitute for laboratory data.
\end{abstract}

\section{Introduction}

Given the challenges such as climate change and the fossil energy crisis, renewable energy production has become much more vital [1-3]. Photovoltaic power production has gained more attention and increased each year because of the benefits of plentiful resources and minimal pollution [4-6]. Improving the reliability and accuracy of photovoltaic output power prediction is an excellent approach $[7,8]$. Accurate and consistent prediction results may assist the power grid in improving power quality and reducing system reserve capacities [9]. However, due to climate change, severe weather events have become more common in recent years, making it challenging to construct an accurate and reliable prediction model [10-12].
Several photovoltaic output power predictive models, including the time series model $[13,14]$, physical model [15], and artificial intelligence model [16, 17], have lately been proposed. The precision of the physical model prediction is heavily reliant on the accuracy of the numerical weather forecast. However, improving NWP accuracy is challenging at the moment [18]. The nonlinear properties of photovoltaic output power cannot be represented using a time series model. As a result, the prediction accuracy is low. The artificial intelligence model is capable of nonlinear fitting [19].

There are essentially three types of hybrid models for predicting photovoltaic output power. The first type predicts photovoltaic output power using an AI model paired with an 
optimization technique [20-23]. Photovoltaic output power was effectively estimated using an upper lower limit approximation and ELMs, as proved by $\mathrm{Ni}$ et al. [20]. According to work done by Liu and his colleagues, few investigations have assessed the uncertainty of predicting photovoltaic power outputs [24]. As a result, several neural networks paired with genetic algorithms were created [25]. The combined model has greater prediction accuracy and reliability, according to empirical data. Using a backpropagation neural platform with the evolving mental technique, Wang and Shen proposed a mixed framework that may be used in many situations [26]. The modeling revealed that the hybrid model outperformed the other methods in terms of predicting photovoltaic output power. While the first type produced acceptable predictive performance, it is challenging to enhance the accuracy further. The reason for this is that the first type did not extract various characteristics of photovoltaic output power. The second type is proposed to overcome this issue. First, the photovoltaic output power is decomposed into its constituent parts using a signal disintegration method. As a result, many characteristics may be retrieved. After that, an artificial intelligence model was created for the prediction of these elements. Wavelet deconstruction and minimal squares support vector systems were used by Giorgi and his colleagues to forecast photovoltaic production potential [27]. In addition, a thorough error assessment was performed to compare the model's efficiency to that of competing models. To forecast photovoltaic output strength, Wang and his colleagues used a wavelet transformation paired with a definitive method [28]. A significant ability to enhance prediction reliability was shown numerically by the recommended technique. WT was utilized by Malvoni and his colleagues to deconstruct the historical data [29]. LSSVM and the group technique were then used to predict photovoltaic output power. Majumder and his colleagues proposed a more reliable photovoltaic output power predictive model for different weathers and times [30]. The prediction model was used in conjunction with altering mode decomposition as well as an extreme learning machine. Even though the signal decomposition model was effectively employed for extracting features, the single artificial intelligence model has its disadvantages. Variables are assigned haphazardly and are prone to falling into local optimum. It is difficult for the 2 nd type to enhance the projection any further. The third type has been proposed to address the issues mentioned above. To begin, the signal decomposition model is utilized on the original photovoltaic output power. Subsequently, for prediction, an artificial intelligence model mixed with an optimization method is constructed. Lin and Pai used seasonal decomposition to manage the initial photovoltaic output power [31]. Subsequently, evolutionary algorithm-optimized minimal squares support vector regression was introduced for forecasting purposes. The suggested model outperformed the competition in terms of predicting accuracy, according to empirical data. Prediction is challenging due to the significant volatility of photovoltaic production power. A new approach based on enhanced experimental deconstruction modeling as well as support vector regression utilizing an improvement approach was developed to solve this problem by Shang and Wei [32]. WT was utilized by Eseye and his colleagues to divide the original photovoltaic output power into finer elements [33]. Next, the regression of the support vector was used. Particle swarm optimization was used to enhance the variables of regression of support vector to increase predicting accuracy. The findings revealed that the hybrid model was more accurate. An ELM improved via the sine cosine approach was utilized by Behera and Nayak to disassemble and anticipate the initial photovoltaic output power employing experimental pattern disintegration [34]. The findings revealed that the suggested model worked well in terms of predicting photovoltaic output power. Even though the third type produces superior predicting results, it nevertheless has the following disadvantages. Since photovoltaic output power has high fluctuation and changeability, no constant-variable simulation can provide a reliable estimation. The versatility of existing prediction models is sometimes overlooked. Secondly, the present decomposition models have not efficiently recovered distinct information from photovoltaic output power. The purpose of writing this article is to propose a model for estimating photovoltaic (PV) output power with higher accuracy compared to previous works. Therefore, the GWO-SVM model was studied, and the performance of this model was estimated by examining the related statistical analyses.

\section{Support Vector Machine}

When it comes to identifying patterns and analyzing data, SVM is one of the monitored training approaches established employing numerical modeling principles [35, 36]. Additionally, statistical analysis and categorization are performed using this parameter [37]. The purpose of our study is to use this technique as a regression approach by employing a nonlinear component of $\Phi(x)$ to move information from a high-dimensional environment to a firstdimensional one [38-40]. The aforementioned nonlinear mapping is accomplished by the creation of the appropriate kernel component of $K\left(x_{i}, y_{i}\right)$ [41]. Additionally, it is considered that certain points are not categorized adequately by a hyperplane; hence, the slack variable is used for this problem [42]. Using $m$ data points in the data space as well as a training dataset of $D=\left\{\left(x_{i}, y_{i}\right) \mid i=1,2,3, \cdots, m\right\}$, a regression function may be presented with $y=w^{T} \Phi(x)+b$, where $\Phi(x)$ represents nonlinear topography function and $b$ and $w$ indicate offsets as well as weight vectors, respectively $[43,44]$. As a result, the optimal formula for the support vector regression model is as follows $[45,46]$ :

$$
\begin{gathered}
\min \frac{1}{2}\|w\|^{2}+c \sum_{i=1}^{m}\left(\xi_{i}+\xi_{i}^{*}\right) \\
\text { s.t. }\left\{\begin{array}{c}
y_{i}-w^{T} . \Phi\left(x_{i}\right)-b \leq \varepsilon+\xi_{i} \\
w^{T} \cdot \Phi\left(x_{i}\right)+b-y_{i} \leq \varepsilon+\xi_{i}^{*} \\
\xi_{i} \geq 0, \xi_{i}^{*} \geq 0
\end{array}\right\},
\end{gathered}
$$


where $C$ is the penalty parameter, $\varepsilon$ displays the loss function variable, and $\xi_{i}^{*}$ and $\xi_{i}$ are the slack parameters [47]. The model loss is calculated once the decisive fault between anticipated and actual scores is more extensive [48, 49]. This problem foundation relates to convex quadratic programming. The Lagrangian function is utilized to integrate the constraint into the cost function, and the dual question may be dissolved in the following manner [50]:

$$
\begin{gathered}
\max \left[-\frac{1}{2} \sum_{i=1}^{m} \sum_{j=1}^{m}\left(\alpha_{i}-\alpha_{i}^{*}\right)\left(\alpha_{j}-\alpha_{j}^{*}\right) \Phi\left(x_{i}\right) \cdot \Phi\left(x_{j}\right)+\sum_{i=1}^{m}\left(\alpha_{i}-\alpha_{i}^{*}\right) y_{i}-\sum_{i=1}^{m}\left(\alpha_{i}-\alpha_{i}^{*}\right) y_{i} \varepsilon\right] \\
\text { s.t. } \sum_{i=1}^{m}\left(\alpha_{i}-\alpha_{i}^{*}\right)=0,0 \leq \alpha_{i} \leq C, 0 \leq \alpha_{i}^{*} \leq C .
\end{gathered}
$$

Here, $\alpha$ indicates the Lagrangian multiplier, and the kernel function computes the movement relation of the used data collection [51]. The function of kernel radial foundation is utilized in this study as [52]

$$
K\left(x_{i}, x_{j}\right)=\exp \left(-\gamma\left\|x_{i}-x_{j}\right\|^{2}\right)
$$

where $\gamma$ denotes the RBF variable. Based on the above explanations, there are two deciding factors in this learning, notably forfeit variable $C$ and the RBF variable, which refers to generalization capacity and estimation performance, respectively. Lastly, the SVM hyperparameters must be optimized $[53,54]$.

\section{Gray-Wolf Optimization}

The GWO algorithm is among the optimization algorithms generated from simulations of gray wolf social hierarchy and predation behavior $[55,56]$. The gray wolf pack has a solid social structure, shown in a pyramidal hierarchy [57]. The gray wolf pack is divided into four categories depending on rank. The low-level wolf follows the high-level wolf in such grades. The gray wolf pack is responsible for hunting actions such as aggressiveness, encirclement, and prey capture. The wolf pack explored for their predation once GWO had found the optimum option [58]. After that, it rummages for the optimum choice based on the gray wolf performance score as well as the relationship among the different levels $[59,60]$.

In the training phase, 70 percent of the records have been employed, with the remainder 30 percent being used to assess the generalizability of the algorithm. All data were normalized between -1 and 1 and put into the SVM model [61].

\section{Sensitivity Analysis}

To examine the effects of the input parameters on the outputs, a mathematical approach known as sensitivity analysis was used [62]. There are many different uses for SA, including determining research priorities, detecting technological flaws, and identifying essential regions [39, 63]. There are two types of SA analyses: global and local [64]. Assuming other factors stay unchanged, local sensitivity examines the impact of one factor on the objective. On the other hand, global sensitivity is a common approach that investigates the influence of inputs on the target once all parameters are changed. Figure 1 shows the efficacy of the input parameters in GWO-SVM for predicting photovoltaic output power. As can be seen, the air temperature has the most significant influence on the photovoltaic output power. The results show that all defined inputs have a considerable impact on the photovoltaic output power values.

\section{Designing a GWO-SVM Model}

Based on previous discussions, $C$, $\varepsilon$, and $\gamma$ control the performance of the SVM algorithm. As a result, GWO was employed to improve these variables in the current investigation. The GWO is divided into four sections: tracking, social hierarchy, encircling, and attacking prey. For modeling wolf hierarchy, four types of gray wolves, namely, alpha $(\alpha)$, beta $(\beta)$, delta ( $\delta$ ), and omega $(\omega)$, are employed with $\alpha, \beta, \delta$, and $\omega$ as solutions. The $a, b$, and $d$ scores are computed according to the associated fitness values so that the top three strategies may predict the prey's location. The literature has detailed information detailing all elements of this method. The GWO is terminated once the last condition is met.

\section{Outlier Analysis}

Outlier diagnosis is a critical statistical method utilized to distinguish sets of data from a larger data collection [65]. Outliers are detected using an efficient technique, termed leverage statistics [66]. The crucial leverage extent $H^{*}$ Hat indicators $(H)$ and standard $(R)$ were all taken into account in the current technique. The Hat index is written as follows $[65,67]$ :

$$
H=X\left(X^{t} X\right)^{-1} X^{t}
$$

where $X$ and $t$ represent the two-dimensional $n \times k$ matrix and the transpose matrix symbol, respectively. The primary oblique of $H$ is where the most likely Hat decision lies in this issue [68]. The presentation of the Williams plot identifies the outliers. The correlation between normalized residue and the Hat indicator is shown in this chart $[69,70]$. The valid range of data is specified as a squared area with a range of \pm 3 standard 


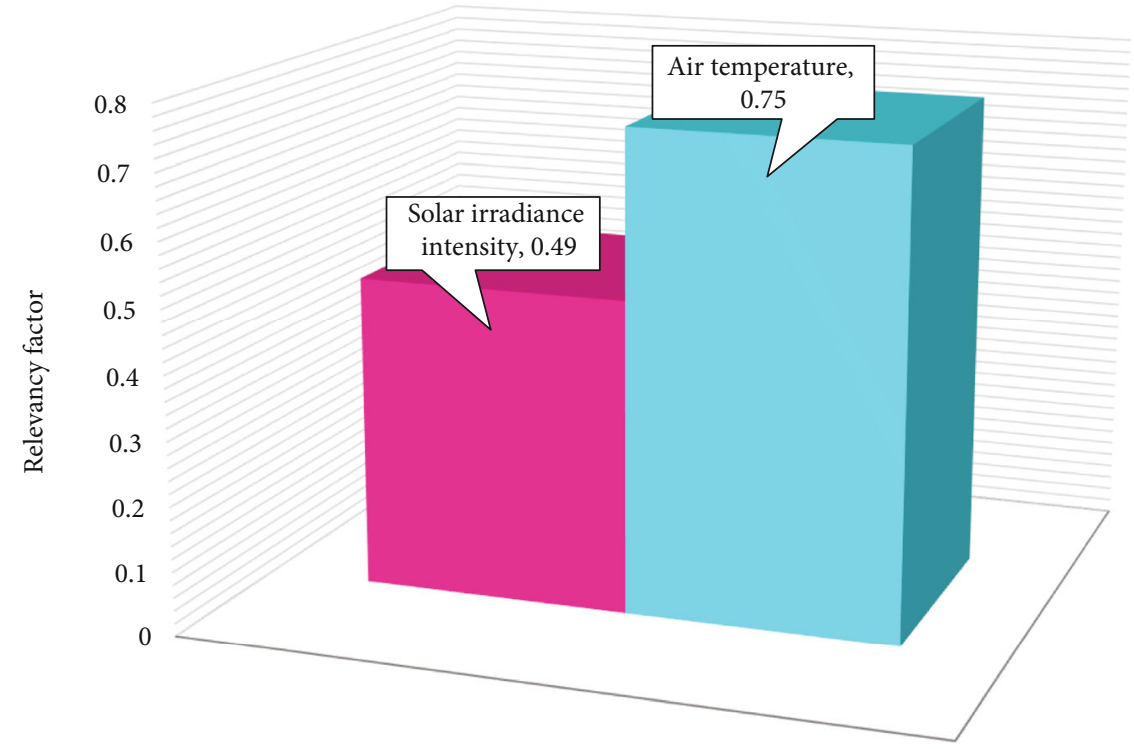

Figure 1: Sensitivity analysis on parameters affecting output.

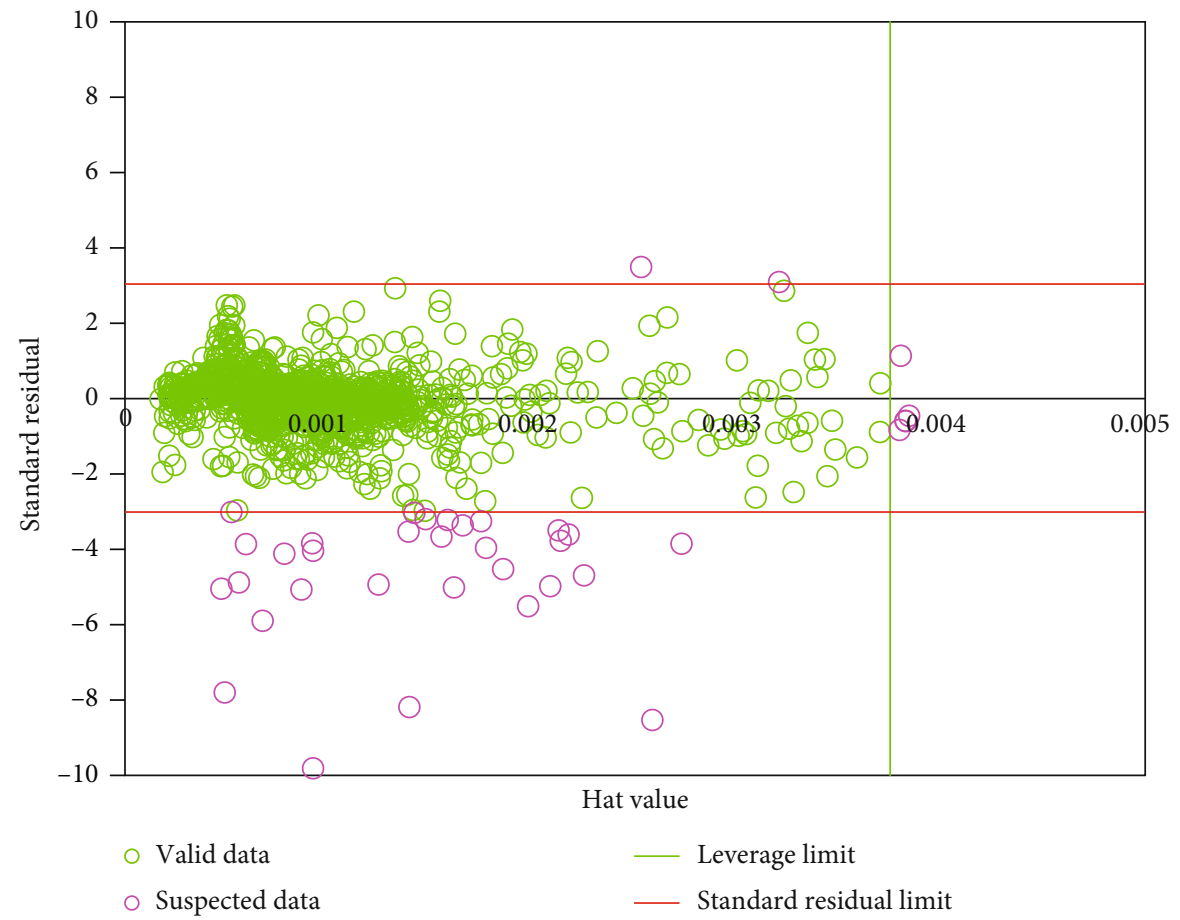

Figure 2: Williams plot and analysis of data to determine suspicious points.

deviations and a strength domain of $3 n /(p+1)(p$ and $n$ indicate the number of inputs of the model and the learning nodes). The significant frequency of data put in the spectra of $-3 \leq R \leq 3$ and $0 \leq H \leq H^{*}$ reveals that GWO-SVM may be used in a wide range of domains. Outliers are described as data $(R$ and $H)$ that exceed the ranges $[-3,3]$ and $\left[0, H^{*}\right]$. The Williams plot of GWO-SVM outputs is depicted in Figure 2. Except for one node in the spectrum of $R<-3$, most photovoltaic output power values investigated in this research fell within the domain of $\left[0, H^{*}\right]$ and $[-3,3]$, demonstrating that the GWO-SVM algorithm is impressive in statistical anal- ysis and may also enhance the capacity to portray the internal relations among the photovoltaic output power score and inputs.

\section{Model Evaluation}

Figure 3 shows the photovoltaic output power value calculated using the GWO-SVM method. The acquired photovoltaic output power values are presented vs. the data index, showing the training and testing results. As can be observed, the suggested model has a high prediction capacity. 


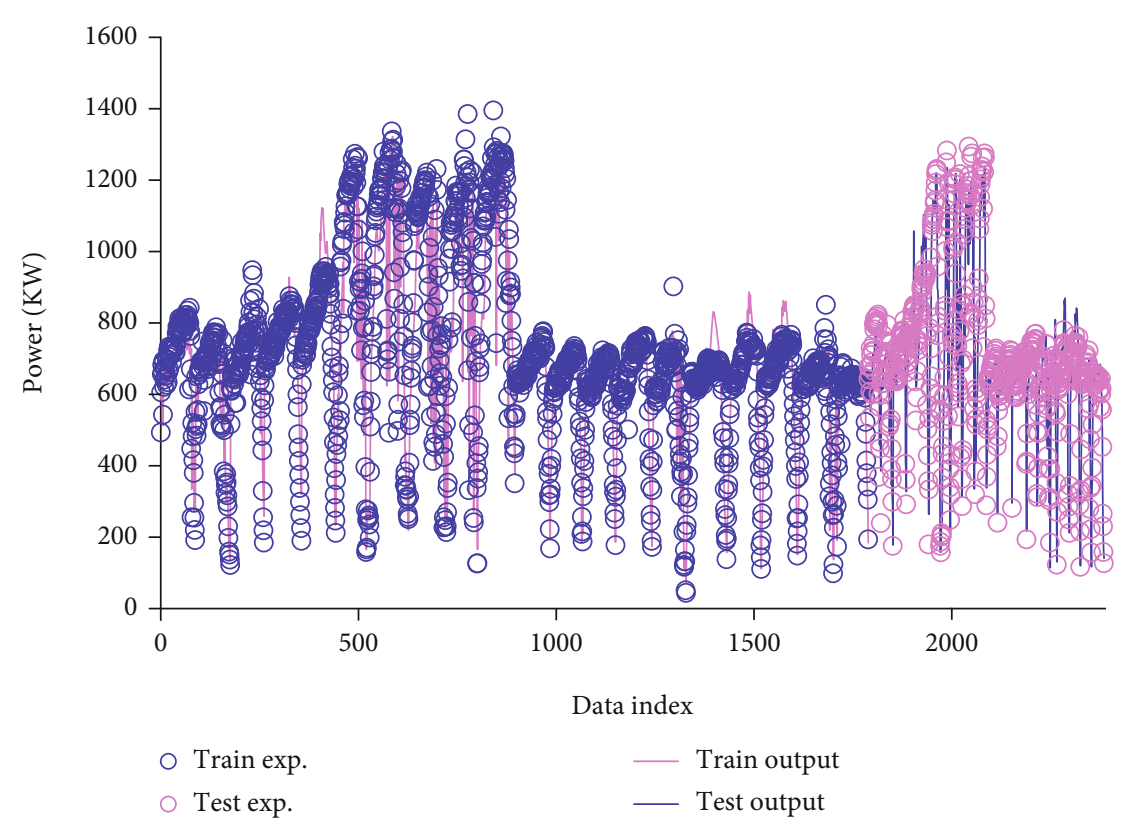

FIGURE 3: Simultaneous observation of predicted and actual values of the target parameter.

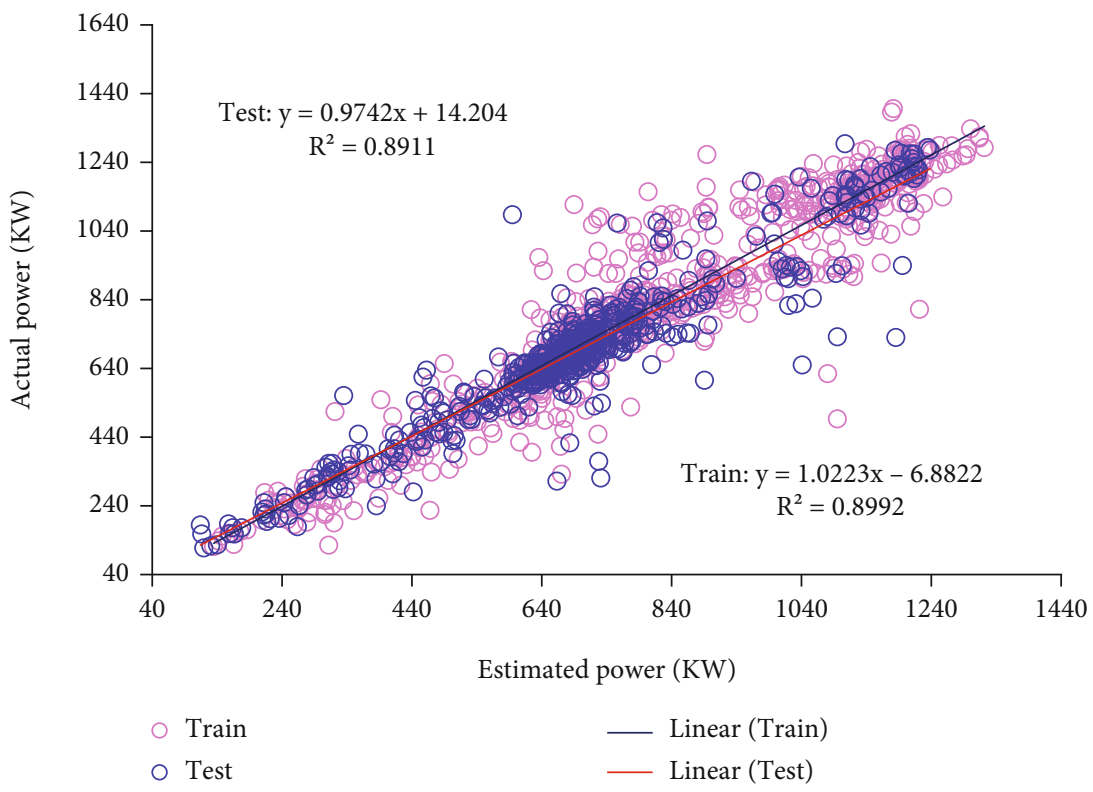

FiguRE 4: Regression analysis performed on the proposed model.

The determination coefficient $\left(R^{2}\right)$ indicates how close determined values are to actual values [44]. $R^{2}$ is a number that ranges between 0 and 1.0. The model predicts more correctly as this parameter approaches unity. The created model's near-unity $R^{2}$ reflects its ability to estimate the photovoltaic output power value. The $R^{2}$ coefficients for the learning and evaluation components of the GWO-SVM algorithms are 0.913 and 0.891 , correspondingly, as shown in the intersecting graph of modeled and actual scores in Figure 4. There are numerous scores around the bisector path in learning and validation data collection, showing that the GWO-SVM has been correctly computed. The prediction capabilities and precision of the GWO-SVM model are shown in Figure 4.
The relative deviation percentages for the GWO-SVM simulation are also demonstrated in Figure 5. It is shown that the GWO-SVM model has high accuracy, with the determined variation not exceeding the $50 \%$ band.

Table 1 shows the values of different statistical parameters in order to evaluate this model in estimating the target parameter.

In order to compare the accuracy of the model proposed in this paper, with the most accurate models ever suggested to predict this parameter by Zhang et al. in 2020, statistical parameters were used [71]. According to Table 2, it is clear that the model proposed in this paper shows higher accuracy in estimating the target parameter. 


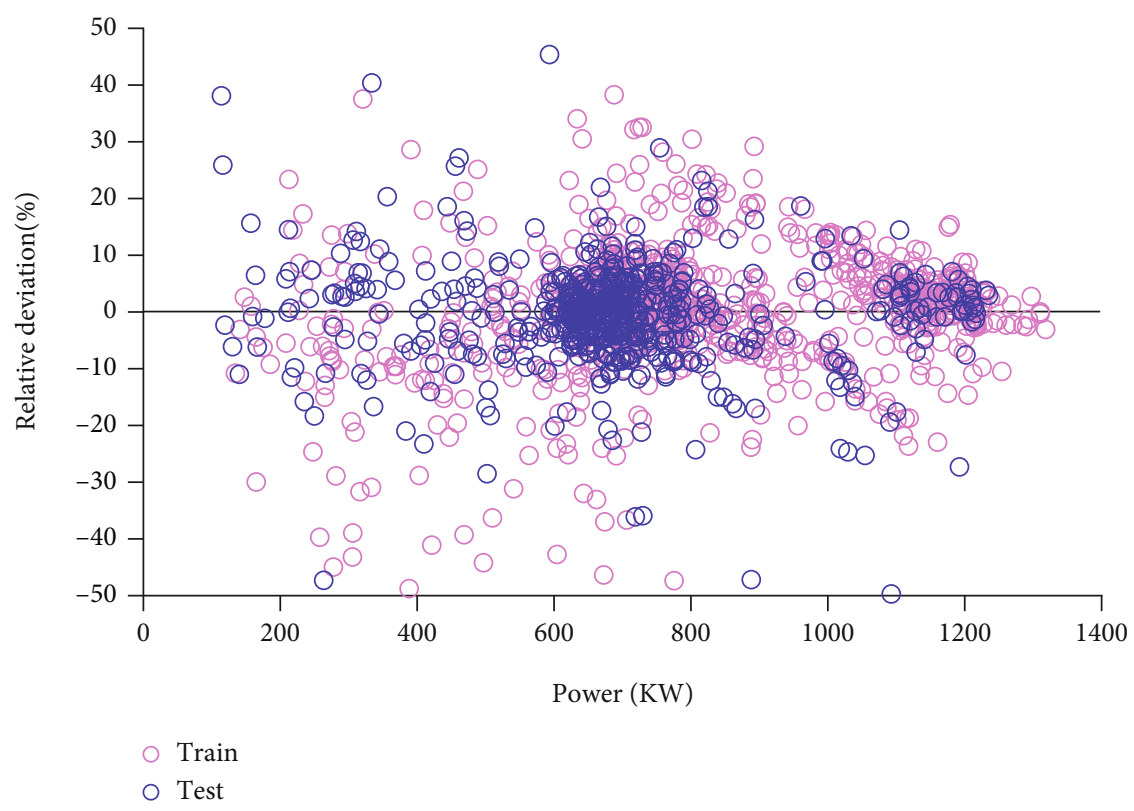

FiguRE 5: Relative deviation values calculated on the model to evaluate its accuracy in predicting target data.

TABLE 1: Values of statistical parameters obtained for the proposed model.

\begin{tabular}{lccccc}
\hline Group & $R^{2}$ & MRE (\%) & MSE & RMSE & STD \\
\hline Train & 0.913 & 7.103 & 5029.275084 & 70.9174 & 53.8453 \\
Test & 0.891 & 7.088 & 5573.878233 & 74.6584 & 59.9669 \\
Total & 0.908 & 7.099 & 5165.425871 & 74.6584 & 55.4307 \\
\hline
\end{tabular}

TABLE 2: Comparing the accuracy of different models in predicting the target parameter.

\begin{tabular}{lc}
\hline Model & RMSE \\
\hline ARIMA & 193.29 \\
LSSVM & 272.51 \\
WNN & 188.30 \\
This work & 74.6584 \\
\hline
\end{tabular}

\section{Conclusions}

This study is aimed at evaluating how effectively a statistical learning-based model may predict the output. For that purpose, the GWO was included in the SVM model. The GWO method performed well when it came to determining tuning parameters. When compared to actual data points, estimations were proved to be highly accurate. The efficiency of the suggested methodologies was established by a definitive agreement between model outputs and absolute values while evaluating the model throughout the training and testing phases, as evidenced by statistical analysis. Comparing the suggested models' results with another reported correlation validated the models' accuracy as expected. In contrast to the robust mathematical methodologies used for this output prediction, the sug- gested strategy for predicting photovoltaic output power is user-friendly, making it a helpful tool for academics, especially in related domains.

\section{Data Availability}

Data references are described in the text of the article.

\section{Conflicts of Interest}

The authors declare that they have no conflicts of interest.

\section{Acknowledgments}

This work was supported by the National key research and development program (2018YFB1500800), Sailing Program of Young Science and Technology Talents supported by Shanghai Science and Technology Commission (Grant No. 19YF1418200), and Young Scientists Fund of the National Natural Science Foundation of China (Grant No. 61902237).

\section{References}

[1] S. R. Sharvini, Z. Z. Noor, C. S. Chong, L. C. Stringer, and R. O. Yusuf, "Energy consumption trends and their linkages with renewable energy policies in east and southeast Asian countries: challenges and opportunities," Sustainable Environment Research, vol. 28, no. 6, pp. 257-266, 2018.

[2] C. Viviescas, L. Lima, F. A. Diuana et al., "Contribution of variable renewable energy to increase energy security in Latin America: complementarity and climate change impacts on wind and solar resources," Renewable and Sustainable Energy Reviews, vol. 113, article 109232, 2019.

[3] U. Bulut and G. Muratoglu, "Renewable energy in Turkey: great potential, low but increasing utilization, and an empirical analysis on renewable energy-growth nexus," Energy Policy, vol. 123, pp. 240-250, 2018. 
[4] B. Lin and J. Zhu, "The role of renewable energy technological innovation on climate change: empirical evidence from China," Science of the Total Environment, vol. 659, pp. 15051512, 2019.

[5] Y. Song, Q. Ji, Y. J. du, and J. B. Geng, "The dynamic dependence of fossil energy, investor sentiment and renewable energy stock markets," Energy Economics, vol. 84, article 104564, 2019.

[6] T. Xia, Q. Ji, D. Zhang, and J. Han, “Asymmetric and extreme influence of energy price changes on renewable energy stock performance," Journal of Cleaner Production, vol. 241, article 118338, 2019.

[7] K. Solaun and E. Cerdá, "Climate change impacts on renewable energy generation. A review of quantitative projections," Renewable and Sustainable Energy Reviews, vol. 116, article 109415, 2019.

[8] N. Vidadili, E. Suleymanov, C. Bulut, and C. Mahmudlu, "Transition to renewable energy and sustainable energy development in Azerbaijan," Renewable and Sustainable Energy Reviews, vol. 80, pp. 1153-1161, 2017.

[9] B. Liu, J. Chen, H. Wang, and Q. Wang, "Renewable energy and material supply risks: a predictive analysis based on an LSTM model," Frontiers in Energy Research, vol. 8, p. 163, 2020.

[10] K. Nam, S. Hwangbo, and C. Yoo, “A deep learning-based forecasting model for renewable energy scenarios to guide sustainable energy policy: a case study of Korea," Renewable and Sustainable Energy Reviews, vol. 122, article 109725, 2020.

[11] M. I. al Irsyad, A. Halog, and R. Nepal, "Renewable energy projections for climate change mitigation: an analysis of uncertainty and errors," Renewable Energy, vol. 130, pp. 536-546, 2019.

[12] B. Wang, Q. Wang, Y. M. Wei, and Z. P. Li, "Role of renewable energy in China's energy security and climate change mitigation: an index decomposition analysis," Renewable and Sustainable Energy Reviews, vol. 90, pp. 187-194, 2018.

[13] L. Martín, L. F. Zarzalejo, J. Polo, A. Navarro, R. Marchante, and M. Cony, "Prediction of global solar irradiance based on time series analysis: application to solar thermal power plants energy production planning," Solar Energy, vol. 84, no. 10, pp. 1772-1781, 2010.

[14] Y. Li, Y. Su, and L. Shu, "An ARMAX model for forecasting the power output of a grid connected photovoltaic system," Renewable Energy, vol. 66, pp. 78-89, 2014.

[15] M. Pierro, M. de Felice, E. Maggioni et al., "Data-driven upscaling methods for regional photovoltaic power estimation and forecast using satellite and numerical weather prediction data," Solar Energy, vol. 158, pp. 1026-1038, 2017.

[16] H. T. Pedro and C. F. Coimbra, "Assessment of forecasting techniques for solar power production with no exogenous inputs," Solar Energy, vol. 86, no. 7, pp. 2017-2028, 2012.

[17] M. Hossain, S. Mekhilef, M. Danesh, L. Olatomiwa, and S. Shamshirband, "Application of extreme learning machine for short term output power forecasting of three gridconnected PV systems," Journal of Cleaner Production, vol. 167, pp. 395-405, 2017.

[18] K. Wang, X. Qi, and H. Liu, “A comparison of day-ahead photovoltaic power forecasting models based on deep learning neural network," Applied Energy, vol. 251, article 113315, 2019.
[19] F. Rodríguez, A. Fleetwood, A. Galarza, and L. Fontán, "Predicting solar energy generation through artificial neural networks using weather forecasts for microgrid control," Renewable Energy, vol. 126, pp. 855-864, 2018.

[20] Q. Ni, S. Zhuang, H. Sheng, G. Kang, and J. Xiao, “An ensemble prediction intervals approach for short-term PV power forecasting," Solar Energy, vol. 155, pp. 1072-1083, 2017.

[21] W. VanDeventer, E. Jamei, G. S. Thirunavukkarasu et al., "Short-term PV power forecasting using hybrid GASVM technique," Renewable Energy, vol. 140, pp. 367-379, 2019.

[22] U. K. Das, K. S. Tey, M. Seyedmahmoudian et al., "Forecasting of photovoltaic power generation and model optimization: a review," Renewable and Sustainable Energy Reviews, vol. 81, pp. 912-928, 2018.

[23] M. Seyedmahmoudian, E. Jamei, G. Thirunavukkarasu et al., "Short-term forecasting of the output power of a buildingintegrated photovoltaic system using a metaheuristic approach," Energies, vol. 11, no. 5, p. 1260, 2018.

[24] L. Liu, Y. Zhao, D. Chang et al., "Prediction of short-term PV power output and uncertainty analysis," Applied Energy, vol. 228, pp. 700-711, 2018.

[25] L.-L. Li, S. Y. Wen, M. L. Tseng, and C. S. Wang, "Renewable energy prediction: a novel short-term prediction model of photovoltaic output power," Journal of Cleaner Production, vol. 228, pp. 359-375, 2019.

[26] H. Wang and J. Shen, "An improved model combining evolutionary algorithm and neural networks for PV maximum power point tracking," IEEE Access, vol. 7, pp. 2823-2827, 2019.

[27] M. G. De Giorgi, P. M. Congedo, M. Malvoni, and D. Laforgia, "Error analysis of hybrid photovoltaic power forecasting models: a case study of Mediterranean climate," Energy Conversion and Management, vol. 100, pp. 117-130, 2015.

[28] H. Wang, H. Yi, J. Peng et al., "Deterministic and probabilistic forecasting of photovoltaic power based on deep convolutional neural network," Energy Conversion and Management, vol. 153, pp. 409-422, 2017.

[29] M. Malvoni, M. G. De Giorgi, and P. M. Congedo, "Forecasting of PV power generation using weather input datapreprocessing techniques," Energy Procedia, vol. 126, pp. 651-658, 2017.

[30] I. Majumder, P. Dash, and R. Bisoi, "Variational mode decomposition based low rank robust kernel extreme learning machine for solar irradiation forecasting," Energy Conversion and Management, vol. 171, pp. 787-806, 2018.

[31] K.-P. Lin and P.-F. Pai, "Solar power output forecasting using evolutionary seasonal decomposition least-square support vector regression," Journal of Cleaner Production, vol. 134, pp. 456-462, 2016.

[32] C. Shang and P. Wei, "Enhanced support vector regression based forecast engine to predict solar power output," Renewable Energy, vol. 127, pp. 269-283, 2018.

[33] A. T. Eseye, J. Zhang, and D. Zheng, "Short-term photovoltaic solar power forecasting using a hybrid wavelet-PSO-SVM model based on SCADA and meteorological information," Renewable Energy, vol. 118, pp. 357-367, 2018.

[34] M. K. Behera and N. Nayak, "A comparative study on shortterm PV power forecasting using decomposition based optimized extreme learning machine algorithm," Engineering Science and Technology, an International Journal, vol. 23, no. 1, pp. 156-167, 2020. 
[35] D. A. Pisner and D. M. Schnyer, "Support vector machine," in Machine Learning, pp. 101-121, Elsevier, 2020.

[36] S. Muthukrishnan et al., "Support vector machine for modelling and simulation of heat exchangers," Thermal Science, vol. 24, pp. 499-503, 2020.

[37] V. Vapnik, The Nature of Statistical Learning Theory Neural Networks, Springer Science \& Business Media, New York, NY, 2013.

[38] A. Baghban, F. Pourfayaz, M. H. Ahmadi, A. Kasaeian, S. M. Pourkiaei, and G. Lorenzini, "Connectionist intelligent model estimates of convective heat transfer coefficient of nanofluids in circular cross-sectional channels," Journal of Thermal Analysis and Calorimetry, vol. 132, no. 2, pp. 1213-1239, 2018.

[39] A. Baghban, M. Kahani, M. A. Nazari, M. H. Ahmadi, and W. M. Yan, "Sensitivity analysis and application of machine learning methods to predict the heat transfer performance of CNT/water nanofluid flows through coils," International Journal of Heat and Mass Transfer, vol. 128, pp. 825-835, 2019.

[40] M. H. Ahmadi, A. Baghban, M. Sadeghzadeh et al., "Evaluation of electrical efficiency of photovoltaic thermal solar collector," Engineering Applications of Computational Fluid Mechanics, vol. 14, no. 1, pp. 545-565, 2020.

[41] S. Abdollahi, H. R. Pourghasemi, G. A. Ghanbarian, and R. Safaeian, "Prioritization of effective factors in the occurrence of land subsidence and its susceptibility mapping using an SVM model and their different kernel functions," Bulletin of Engineering Geology and the Environment, vol. 78, no. 6, pp. 4017-4034, 2019.

[42] Y. Zhou, F. J. Chang, L. C. Chang, I. F. Kao, Y. S. Wang, and C. C. Kang, "Multi-output support vector machine for regional multi-step-ahead PM2. 5 forecasting," Science of the Total Environment, vol. 651, pp. 230-240, 2019.

[43] A. Zendehboudi, M. A. Baseer, and R. Saidur, "Application of support vector machine models for forecasting solar and wind energy resources: a review," Journal of Cleaner Production, vol. 199, pp. 272-285, 2018.

[44] D. Ahangari, R. Daneshfar, M. Zakeri, S. Ashoori, and B. S. Soulgani, "On the prediction of geochemical parameters (TOC, S1 and S2) by considering well log parameters using ANFIS and LSSVM strategies," Petroleum, 2021.

[45] S. Huang, N. Cai, P. P. Pacheco, S. Narrandes, Y. Wang, and $\mathrm{W}$. Xu, "Applications of support vector machine (SVM) learning in cancer genomics," Cancer Genomics \& Proteomics, vol. 15, no. 1, pp. 41-51, 2018.

[46] J. Cervantes, F. Garcia-Lamont, L. Rodríguez-Mazahua, and A. Lopez, "A comprehensive survey on support vector machine classification: applications, challenges and trends," Neurocomputing, vol. 408, pp. 189-215, 2020.

[47] N. A. Almansour, H. F. Syed, N. R. Khayat et al., "Neural network and support vector machine for the prediction of chronic kidney disease: a comparative study," Computers in Biology and Medicine, vol. 109, pp. 101-111, 2019.

[48] H. Wang, B. Zheng, S. W. Yoon, and H. S. Ko, “A support vector machine-based ensemble algorithm for breast cancer diagnosis," European Journal of Operational Research, vol. 267, no. 2, pp. 687-699, 2018.

[49] S. M. Alizadeh, I. Alruyemi, R. Daneshfar, M. MohammadiKhanaposhtani, and M. Naseri, "An insight into the estimation of drilling fluid density at HPHT condition using PSO-, ICA-, and GA-LSSVM strategies," Scientific Reports, vol. 11, no. 1, pp. 1-14, 2021.
[50] L. Hu and J. Cui, "Digital image recognition based on fractional-order-PCA-SVM coupling algorithm," Measurement, vol. 145, pp. 150-159, 2019.

[51] M. Zamen, A. Baghban, S. M. Pourkiaei, and M. H. Ahmadi, "Optimization methods using artificial intelligence algorithms to estimate thermal efficiency ofPV/T system," Energy Science \& Engineering, vol. 7, no. 3, pp. 821-834, 2019.

[52] Z. Shao, S. L. Yang, F. Gao, K. L. Zhou, and P. Lin, "A new electricity price prediction strategy using mutual informationbased SVM-RFE classification," Renewable and Sustainable Energy Reviews, vol. 70, pp. 330-341, 2017.

[53] M. U. Ali, H. F. Khan, M. Masud, K. D. Kallu, and A. Zafar, “A machine learning framework to identify the hotspot in photovoltaic module using infrared thermography," Solar Energy, vol. 208, pp. 643-651, 2020.

[54] A. Baghban, J. Sasanipour, F. Pourfayaz et al., "Towards experimental and modeling study of heat transfer performance of water- $\mathrm{SiO}_{2}$ nanofluid in quadrangular cross-section channels," Engineering applications of computational fluid mechanics, vol. 13, no. 1, pp. 453-469, 2019.

[55] X. Xue, Y. Zheng, and C. Lu, "Optimal allocation of distributed energy supply system under uncertainty based improved gray wolf algorithm," Distributed Generation \& Alternative Energy Journal, vol. 37, pp. 381-400, 2022.

[56] C. P. Igiri, Y. Singh, and R. C. Poonia, "A review study of modified swarm intelligence: particle swarm optimization, firefly, bat and gray wolf optimizer algorithms," Recent Advances in Computer Science and Communications, vol. 13, no. 1, pp. 5-12, 2020.

[57] E. Emary, H. M. Zawbaa, and A. E. Hassanien, "Binary grey wolf optimization approaches for feature selection," Neurocomputing, vol. 172, pp. 371-381, 2016.

[58] K. Qian, X. Liu, Y. Wang, X. Yu, and B. Huang, "Modified dual extended Kalman filters for SOC estimation and online parameter identification of lithium-ion battery via modified gray wolf optimizer," Proceedings of the Institution of Mechanical Engineers Part D: Journal of Automobile Engineering, no. article 0954407021104669, 2021.

[59] L. Sun, C. Tang, M. Xu, and Z. Lei, "Sub-pixel displacement measurement based on the combination of a gray wolf optimizer and gradient algorithm," Applied Optics, vol. 60, no. 4, pp. 901-911, 2021.

[60] J. Li and F. Yang, "Task assignment strategy for multi-robot based on improved grey wolf optimizer," Journal of Ambient Intelligence and Humanized Computing, vol. 11, no. 12, pp. 6319-6335, 2020.

[61] B. S. Yıldız and A. R. Yıldız, "Comparison of grey wolf, whale, water cycle, ant lion and sine-cosine algorithms for the optimization of a vehicle engine connecting rod," Materials Testing, vol. 60, no. 3, pp. 311-315, 2018.

[62] N. Kardani, A. Zhou, M. Nazem, and X. Lin, "Modelling of municipal solid waste gasification using an optimised ensemble soft computing model," Fuel, vol. 289, article 119903, 2021.

[63] A. Baghban, M. N. Kardani, and A. H. Mohammadi, "Improved estimation of Cetane number of fatty acid methyl esters (FAMEs) based biodiesels using TLBO-NN and PSONN models," Fuel, vol. 232, pp. 620-631, 2018.

[64] N. Kardani, A. Bardhan, P. Samui, M. Nazem, A. Zhou, and D. J. Armaghani, "A novel technique based on the improved firefly algorithm coupled with extreme learning machine (ELM-IFF) for predicting the thermal conductivity of soil," Engineering with Computers, pp. 1-20, 2021. 
[65] A. Ghanbari, M. N. Kardani, A. Moazami Goodarzi, M. Janghorban Lariche, and A. Baghban, "Neural computing approach for estimation of natural gas dew point temperature in glycol dehydration plant," International Journal of Ambient Energy, vol. 41, no. 7, pp. 775-782, 2020.

[66] A. Baghban and M. Adelizadeh, "On the determination of cetane number of hydrocarbons and oxygenates using adaptive neuro fuzzy inference system optimized with evolutionary algorithms," Fuel, vol. 230, pp. 344-354, 2018.

[67] A. Lekomtsev, A. Keykhosravi, M. B. Moghaddam, R. Daneshfar, and O. Rezvanjou, "On the prediction of filtration volume of drilling fluids containing different types of nanoparticles by ELM and PSO-LSSVM based models," Petroleum, 2021.

[68] M. N. Kardani, A. Baghban, J. Sasanipour, A. H. Mohammadi, and S. Habibzadeh, "Group contribution methods for estimating $\mathrm{CO}_{2}$ absorption capacities of imidazolium and ammonium-based polyionic liquids," Journal of Cleaner Production, vol. 203, pp. 601-618, 2018.

[69] F. Mousazadeh, M. H. T. Naeem, R. Daneshfar, B. S. Soulgani, and M. Naseri, "Predicting the condensate viscosity near the wellbore by ELM and ANFIS-PSO strategies," Journal of Petroleum Science and Engineering, vol. 204, article 108708, 2021.

[70] R. Setiawan, R. Daneshfar, O. Rezvanjou, S. Ashoori, and M. Naseri, "Surface tension of binary mixtures containing environmentally friendly ionic liquids: insights from artificial intelligence," Environment, Development and Sustainability, vol. 23, no. 12, pp. 17606-17627, 2021.

[71] J. Zhang, Z. Tan, and Y. Wei, "An adaptive hybrid model for day-ahead photovoltaic output power prediction," Journal of Cleaner Production, vol. 244, article 118858, 2020. 